\title{
Extreme Frühchen: bessere Überlebenschancen bei Intention zu aktiver Behandlung
}

\begin{abstract}
Bei den Überlebensraten von Extremfrühgeborenen gibt es eine große Varianz. Unterschiedliche Ergebnisse von US-Kliniken scheinen mit lebenserhaltenden Maßnahmen vor der 25. Gestationswoche zusammenzuhängen.
\end{abstract}

Collen Frühgeborene an der Grenze $\checkmark$ der Lebensfähigkeit lebensverlängernde Maßnahmen erhalten? Diese schwierige Entscheidung wird in den meisten Leitlinien vom Gestationsalter abhängig gemacht. Bei Kindern mit Geburt vor der 22. Schwangerschaftswoche (SSW) wird eine aktive Behandlung wegen Aussichtslosigkeit im Allgemeinen nicht empfohlen. Eine Grauzone besteht zwischen der 22. und 24. SSW. Laut deutscher S2-Leitlinie sollen in der 22. bzw. 23. SSW Geborene nur auf ausdrücklichen Wunsch der Eltern bzw. in gemeinsamer Entscheidung mit den Eltern aktiv behandelt werden; erst ab der 24. SSW ist eine lebenserhaltende Therapie „im Regelfall anzustreben“. Eine retrospektive Studie aus den USA zeigt nun, dass Neugeborene mit einem Gestationsalter von 22-24 Wochen öfter überleben, wenn die Intention zu aktiver Behandlung besatnd.

In der Studie wurde das Schicksal von 4.987 vor der 27. SSW in einem spezialisierten Krankenhaus Geborenen bis zum Alter von 18-22 Monaten verfolgt. 4.329 der extremen Frühchen erhielten kurative Maßnahmen (Surfactant-Behandlung, Intubation, Atemunterstützung, parenterale Ernährung, Epinephringaben und/oder Thoraxkompressionen). Palliativ versorgte Kinder waren eher klein für ihr Alter und hatten häufiger einen 1-Minuten-Apgar-Score $<3$. Der Anteil aktiv behandelter Kinder stieg mit dem Geburtsalter von $22,1 \%$ in der 22. auf $71,8 \%$ in der 23 . und $97,1 \%$ in der 24. SSW (in der 25. bzw. 26. SSW: 99,6\% bzw. 99,8\%). Bei den Jüngsten war der Einsatz lebenserhaltender Therapien mit einer erheblich besseren Prognose assoziiert: In der 22. SSW Geborene hatten eine Überlebensrate von 5,1\% (3,4\% überlebten ohne schwere, $2,0 \%$ ohne mittelschwere Behinderungen). Bei den ebenso früh Geborenen mit lebenserhaltender Therapie betrugen die Quoten
23,1\%, 15,4\% und 9,0\%. Diese Beziehung schwächte sich mit dem Geburtsalter ab. Der Vergleich der 24 teilnehmenden Krankenhäuser ergab vor allem bei den in der 22. und 23. SSW Geborenen eine große Schwankungsbreite im Einsatz kurativer Maßnahmen (8-100\% bzw. 5397\%). Die Unterschiede erklären zum Teil die Varianz in der krankenhausspezifischen Prognose der Kinder (78\% der Schwankungen beim Überleben, 75\% beim Überleben ohne schwere, $41 \%$ beim Überleben ohne mittelschwere Behinderung). Bei in der 24. SSW Geborenen zeigte sich dies abgeschwächt, bei Kindern mit 25 oder 26 SSW lieferte die aktive Behandlung dagegen keine Erklärung für krankenhausabhängige Differenzen. Wieso manche Krankenhäuser öfter, andere seltener lebenserhaltende Maßnahmen initiieren, lässt sich aus der Studie nicht erklären. Wie viele extreme Frühchen in einem Krankenhaus versorgt wurden, hatte auch keinen Einfluss auf die Prognose. Dr. Beate Schumacher

Rysavy MA et al. Between-Hospital Variation in Treatment and Outcomes in Extremely Preterm Infants. N Engl J Med 2015; 372: 1801-11

\section{Kommentar}

Hohe Unterschiede in den Versorgungsund in den Outcome-Raten extrem kleiner Frühgeborener sind in den USA häufig beobachtet worden. Sie korrelieren sehr gut mit europäischen Daten, z.B. aus der Anfang der 2000er-Jahre durchgeführten MOSAICStudie. In europäischen Regionen, wo Grenzen für die Versorgung Frühgeborener z.B. in der $26+0$. SSW gesetzt wurden, war das Outcome dieser Gestationsaltergruppen jeweils schlechter bezüglich Überleben und Überleben ohne starke Behinderungen als in Ländern, in denen die Grenze der aktiven Versorgung tiefer lag.

Erklärt wird dies allgemein durch eine höhere Kompetenz in der Versorgung extrem kleiner Frühgeborener in Zentren, welche die kleinsten Kinder versorgen. Die Behandlungsraten lagen in der vorliegenden Studie zwischen 22,1 \% (7,7-100) der Frühgeborenen in der $22+0$. SSW bis zu 99,8 \% in der $26+0$. SSW. Die Überlebensraten bzw. die Überlebensraten ohne schwere Behinderungen reichen von $5,1 \%(0-10,6)$ Überlebensrate und 3,4 \% intakter Überlebensrate $(0-6,9)$ in der $22+0$. SSW bis zu 81,4 \% Überleben bzw. 75,6 \% Überleben ohne schwere Behinderung in der $26+0$. SSW. Es zeigte sich eine deutliche Abhängigkeit dieser Überlebensrate von der Anzahl aktiver Behandlungen. Das heißt, Unterschiede im praktischen Vorgehen in der Versorgung extrem kleiner Frühgeborener zwischen den Krankenhäusern haben eine direkte Auswirkung auf Überlebensrate und Überlebensrate ohne schwere Behinderungen. Dieser Zentrumseffekt, jedoch nicht aufgeschlüsselt nach der Intention aktiver Behandlung, findet sich auch in Deutschland. Das Journal "neonatal network" als zurzeit größte Frühgeborenendatenbasis der Welt, zeigt bei überwiegend aktivem Vorgehen der deutschen Zentren eine Überlebensrate von $51,8 \%$ in der $22+$ 0 . SSW und von $88,2 \%$ in der $26+0$. SSW, also bei ähnlicher Verteilung höhere Überlebensraten und Überlebensraten ohne schwere Behinderung.

Die Analyse dieser Ergebnisse ist für die Praxis von Perinatalzentren höchster Versorgungsstufe sehr wichtig. Die ethische Frage, ob für die verbesserte Überlebenschance eines Kollektivs der Tod oder auch die schwere Behinderung von Einzelnen in Kauf genommen wird, ist unberührt von dieser wissenschaftlichen Untersuchung. Es muss aber in der perinatologischen Planung berücksichtigt werden, dass die Intention zu aktiver Behandlung im Zusammenhang mit der Überlebensrate steht und dies auch für die Gruppe von Kindern der jeweils reiferen Gestationsalter-Gruppe gilt. Das bedeutet, eine aktive Behandlungsintention in sehr unreifen Gestationsaltern verbessert auch das Outcome in reiferen Gruppen im jeweiligen Perinatalzentrum.

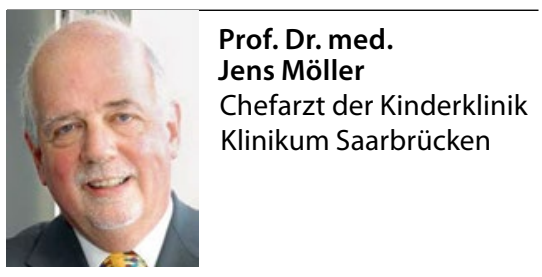

